\title{
Factors related to hypertensive phase after glaucoma drainage device implantation
}

This article was published in the following Dove Press journal:

Clinical Ophthalmology

\author{
Orathai Pitukcheewanont ${ }^{\prime}$ \\ Visanee Tantisevi ${ }^{1}$ \\ Sunee Chansangpetch ${ }^{2}$ \\ Prin Rojanapongpun' \\ 'Department of Ophthalmology, \\ Faculty of Medicine, Chulalongkorn \\ University, Bangkok, Thailand; \\ ${ }^{2}$ Department of Ophthalmology, King \\ Chulalongkorn Memorial Hospital, \\ Thai Red Cross Society, Bangkok, \\ Thailand
}

Background: The aim of the study was to evaluate factors related to hypertensive phase (HP) after glaucoma drainage device (GDD) implantation.

Patients and methods: Retrospective charts review of glaucoma patients who underwent GDD implantation and completed 12 months of follow-up at King Chulalongkorn Memorial Hospital since 2004 was performed. Patients were divided into two groups according to the presence or absence of HP. The data were analyzed for factors associated with HP. The surgical outcomes including surgical failure, post-operative visual acuity (VA), post-operative number of medications, post-operative intraocular pressure (IOP), and post-operative complication were compared between the two groups.

Results: Seventy-two patients were included. Most were diagnosed with secondary glaucoma $(\mathrm{N}=51,70.8 \%)$, and Baerveldt was the most implanted GDD ( $=49,68.1 \%)$. Mean \pm SD of IOP was significantly lower: $27.1 \pm 9.6 \mathrm{mmHg}$ at pre-operation and $13.7 \pm 59 \mathrm{mmHg}$ at 12 -month follow-up $(p<0.001)$. HP was identified in 38/72 patients $(52.8 \%, 95 \%$ CI 40.7-64.7). Risk factor of HP was pre-operative VA equal or better than 20/70 ( $p=0.021$, OR 7.5; 95\% CI 1.4-41.4). Presence of underlying heart disease (patients in this group had taken anti-platelets) was found as a protective factor for HP ( $p=0.027$, OR 0.06 ; $95 \%$ CI $0.00-0.72)$. It was speculated that the antiinflammatory effect of the anti-platelets, particularly aspirin, may be responsible for controlling inflammation and retarding fibrosis formation around drainage implants. Failure rate at 12 months was $24 / 72(33 \%)$. There was no difference in all surgical outcomes between the two groups. Survival analysis also showed no significant difference between HP and non-HP group.

Conclusion: HP commonly occurred after GDD implantation. Pre-operative VA was a risk factor for the development of HP, whereas presence of underlying heart disease was a protective factor. No association between HP and surgical outcomes was identified.

Keywords: glaucoma implant, post-operative intraocular pressure, risk factors, surgical outcomes

\section{Introduction}

Glaucoma, defined by chronic progressive optic neuropathy, is the second leading cause of avoidable blindness worldwide. Intraocular pressure (IOP) is proved to be the main risk factor responsible for changes in optic nerve structure and visual function in glaucoma. Therefore, the primary goal of treatment is to lower IOP. Surgical procedures are required in glaucoma when IOP can not be controlled by topical medications alone.

Glaucoma drainage device (GDD) implantation, one of the surgical interventions, is performed in complicated glaucoma, wherein conventional filtering surgery has failed or likely to fail, such as neovascular glaucoma and uveitic glaucoma. After GDD implantation, post-operative IOP usually undergoes through three phases: hypotensive
Correspondence: Visanee Tantisevi Department of Ophthalmology, Faculty of Medicine, Chulalongkorn University, Ground Floor Chumbhot Building, 1873, Rama 4 Road, Patumwan, Bangkok 10II0, Thailand

Tel +6622564142

Fax +66 22528290

Email tvisanee@gmail.com 
phase that might occur immediately in the first week after surgery and hypertensive phase (HP), known as a period of large IOP rise after glaucoma drainage implantation, usually tends to be noticed within 3 months after surgery, then IOP gradually decreases and becomes stable in steady phase thereafter. During HP, dense fibrous tissue over the plate and intense bleb wall congestion are histologically observed. ${ }^{1,2}$ Since most patients who receive drainage device implantations have advanced glaucoma, such a phase could further damage the optic nerve, requiring more treatments and probably leads to implant failure. ${ }^{3}$

The incidence of HP after GDD implantation from previous reports varies among studies and types of device implanted. Higher incidence of hypertensive phase has been reported in Ahmed device (40\%-80\%) than was the Baerveldt (20-30) or Molteno $(20 \%-30 \%))^{2,4}$ A retrospective study of 85 patients with Ahmed implantation showed that HP usually peaked in the first month after surgery. ${ }^{2}$ Ayyala et al's study revealed that the rate of HP was $83.5 \%$ in Ahmed group and $43.5 \%$ in double-plate Molteno group. ${ }^{5}$ Different incidence was reported by Nouri-Mahdavi and Caprioli, and occurred in $56 \%$ of people in Ahmed group. ${ }^{6}$

Although HP is widely mentioned in Ahmed device, there are few reports of HP in Baerveldt. The Ahmed versus Baerveldt Study study compared the outcomes of Ahmed-FP7 valve and Baerveldt-350 implantation in 238 refractory glaucoma patients. Both study groups revealed noticeable rising of mean post-operative IOP during 2 weeks to 3 months; this might imply their HP albeit the study itself did not mention the term. In the same study, failure rates of $51 \%$ and $34 \%$ were noted in Ahmed group and Baerveldt group, respectively. High IOP was identified as the most common cause of failure, thus emphasizing the importance of this condition. ${ }^{7}$

Despite high incidence of HP, there are few reports of the responsible factors. ${ }^{8,9}$ Furthermore, only little data about effects of such events are published. This study aimed to reveal factors related to HP and mid-term (12 months) HP-related outcomes by reviewing data of glaucoma patients who received GDD implantations at King Chulalongkorn Memorial hospital.

\section{Patients and methods}

This retrospective observational study was approved by the Institutional Review Board (IRB) of the Faculty of Medicine, Chulalongkorn University, IRB number 097/58. According to our IRB regulations, written consent from each individual subject for the retrospective study performed only chart review was not compulsorily required. In terms of using patients' data, the letter to the director of the hospital for permission to use the data was officially accepted.
Confidentiality had to be maintained for all reviewed data. After the approval, the study was performed in accordance with the ethical standards of the Declaration of Helsinki.

Retrospective charts of glaucoma patients aged 20 years and older who underwent GDD implantation between January 2004 and January 2015 and completed the follow-up period of 12 months at King Chulalongkorn Memorial Hospital were reviewed. The patients who had tube obstruction, retraction or valve malfunction were excluded. All GDD implantations were done by glaucoma specialists, and post-operative medications such as anti-glaucoma medications were adjusted according to the patient's glaucoma status.

\section{Surgical procedures}

After patients were informed regarding the details of the surgery rationale and informed consent to surgery was obtained, patients were scheduled to have GDD implantation under local or general anesthesia depending on their condition.

Patients were given local anesthesia in supine position, and sterile draping was used in the usual sterile manner. Topical $0.5 \%$ tetracaine was used before performing radial conjunctival peritomy followed by the incision made along the limbus. Conjunctiva was extensively undermined to expose intermuscular space; 3-5 mL of 2\% Lidocaine without adrenaline was injected along the curve of the eyeball in to the deep sub-Tenon area using a blunt tip cannula attached to a $5 \mathrm{~mL}$ syringe. Intermuscular space at the superotemporal or superonasal quadrant was well prepared for the insertion of the GDD plate.

Either valve or non-valve GDD, tube patency and platevalve were checked with the injection of balanced salt solution before introducing the plate to the prepared area. Ahmed implant was placed underneath the reflected conjunctiva at the prepared quadrant. In cases implanted with Baerveldt, two recti muscles were identified with muscle hook before wings of the plate were inserted into the scleral space under the muscles; the center of the plate was then properly positioned. Double-plate Molteno placement followed the same procedure as followed for Baerveldt. The first plate was placed at the superotemporal or superonasal quadrant and the second plate was placed in the fellow quadrant. Each implant was fixed to the underlying sclera using 6-0 or 8-0 nylon.

In cases with non-valve GDD, the tube was tightly ligated with 6-0 vicryl and tested for its total occlusion before length adjustment and insertion.

Tube length was adjusted before insertion into the anterior chamber through the fistula made by a $23 \mathrm{G}$ needle at $1-2 \mathrm{~mm}$ behind the limbus. Tube tip was in bevel-up fashion. Then, the tube was fixed to the sclera using 8-0 vicryl. Corneo-scleral patch was trimmed into the desired width and proper thickness 
for tube coverage in order to prevent late tube erosions. The patch was fixed to the sclera using 8-0 vicryl. One-to-two slit cuts were done on the side of the tube anteriorly to the ligature site before tube entering anterior chamber. The cuts acted as a vent for aqueous to drain which helped lowering IOP during the early post-operative days despite tight ligature persisted and occluded draining through tube onto the implant plate.

The anterior chamber was filled with balanced salt solution through the side port. This was done to recheck the aqueous flow through tube patency in Ahmed-implanted eyes as well as to check the ligated tube for its aqueous flow occlusion in Baerveldt- or Molteno-implanted eyes. Moreover, any possible leaking sites at the entry of the tube were completely checked.

Conjunctiva was then re-approximated, covering the whole plate, tube and the entire corneo-scleral patch, by $8-0$ vicryl. Topical antibiotics were used for the eye at the end of surgery.

Post-operative anti-glaucoma medication was ceased immediately in Ahmed cases, whereas it was still considered in cases with Baerveldt and Molteno in the first few weeks before suture ligature lysed.

Topical steroid and antibiotics were started immediately after surgery. Steroid was employed every 2 hours in the first few weeks and then tapered off until intraocular inflammation subsided, whereas cessation of topical antibiotics was usually done after 1 week post-operation. Systemic steroid and NSAID were not part of the regular regimen unless indicated by the surgeons.

Figures 1 and 2 show post-operative tube position in uveitic glaucoma patient.

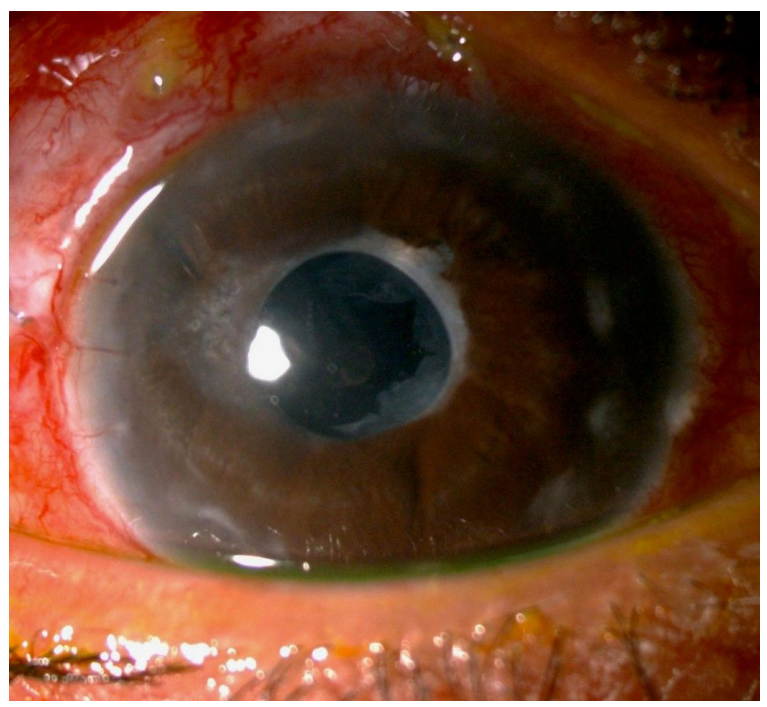

Figure I Post-operative tube position in uveitic glaucoma patient.

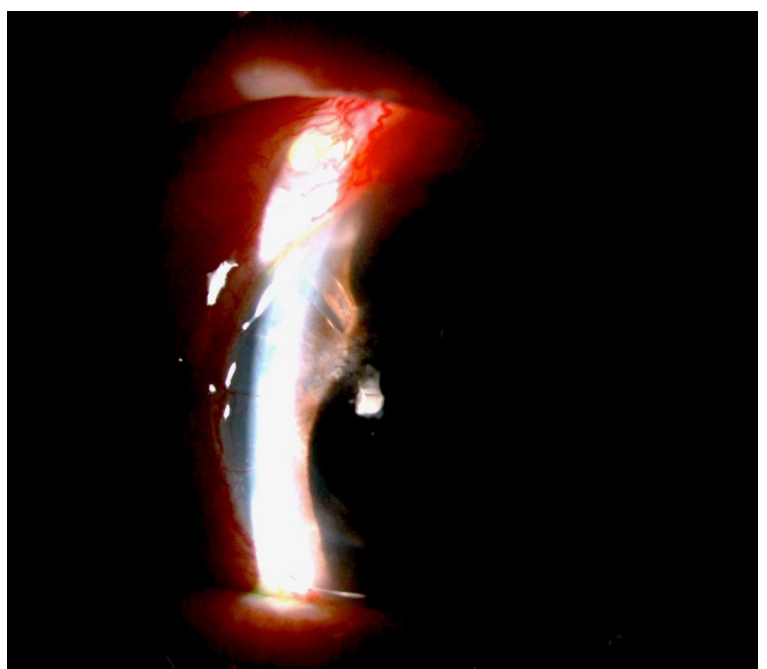

Figure $\mathbf{2}$ Slit beam showing post-operative tube position in uveitic glaucoma patient.

\section{Outcome measurements and definitions}

Baseline characteristics were documented for each patient: age at the time of surgery, sex, underlying disease, eye laterality, glaucoma diagnosis, status of the lens, previous glaucoma surgery, type of GDD, pre-operative visual acuity (VA), pre-operative number of medications and pre-operative IOP. Post-operative VA, IOP, number of medications and complications at 1 day, 2 weeks, 1 month, 2 months, 3 months, 4 months, 6 months, 9 months and 1 year after the surgery were recorded. The subjects were divided into two groups according to the presence (HP) or absence of HP (non-HP). The primary outcome was factors related to HP. The secondary outcomes were rate of HP, relationship of HP and post-operative complications, and surgical failure. One eye of all patients was selected; if both eyes were eligible, computer-generated simple randomization was used.

HP was defined as IOP $>21 \mathrm{mmHg}$ during the first 3 months after surgery with or without medications or IOP $>21 \mathrm{mmHg}$ after reaching 2 consecutive weeks of functional post-operative period, in which IOP was noticeably lower than $22 \mathrm{mmHg}$. All elevated IOP must not be caused by tube obstruction, retraction or valve malfunction.

Treatment failure was defined as 1) IOP $>21 \mathrm{mmHg}$ or IOP reduction $\leq 20 \%$ from baseline for 2 consecutive weeks despite maximally tolerated medications, 2) patients needed further significant surgical intervention at any time point, 3) VA become no light perception.

Any complication that occurred within 3 months after surgery was defined as early complication and complication that occurred after that time was defined as late complication. 


\section{Statistical analysis}

The statistical analysis was performed using SPSS version 22.0 (IBM corp., Armonk, NY, USA). Comparisons between HP and non-HP groups were performed to evaluate factors associated with HP by using $t$-test for continuous data, chisquare test for continuous data, Fisher's exact test and multiple logistic regression. Chi-square test, Fisher's exact test and logistic regression model were used to assess the effects of HP on surgical outcomes. Pre-operative and post-operative surgical outcomes were compared using Wilcoxon signed ranks test and $\mathrm{McNemar}$ test. Comparison of failure and non-failure was done using chi-square test and Fisher's exact test. KaplanMeier method was used for determining survival analysis. $p$-value less than 0.05 was considered statistically significant.

\section{Results}

Retrospective charts review revealed the drainage device code of 113 eyes. Due to some mismatched code registration, some charts were dismissed. The eligible charts of 89 eyes (82 patients) were used for reviewing. Eleven eyes (10 patients) did not fulfill the criteria: nine eyes had an incomplete follow-up period, one eye underwent keratoprosthesis implantation during follow up and one eye had tube obstruction. A total of 72 eyes (72 patients) out of 89 eyes $(80.9 \%)$ met the inclusion criteria.

The mean (SD) age was 54.8 (17.7) years. Most patients were diagnosed with secondary glaucoma (70.8\%) and had at least one prior glaucoma filtering surgery (68.06\%). Mean (SD) vertical cup-disc ratio (CD) in 54 patients was 0.74 (0.23). The missing data were due to corneal edema and media opacity. Most patients had pre-operative VA worse than 20/200 (68.05\%). In this study, 18 Ahmed patients (25\%) had valve GDD; five Molteno (double-plate) patients (6.9\%) and 49 Baerveldt patients (68.06\%) had non-valve GDD. Baseline characteristics are summarized in Table 1.

HP was presented in $38 / 72$ patients $(52.8 \%, 95 \%$ CI 40.7-64.7), and occurred in about half of all types of GDD implantation (Baerveldt 55.1\% [N=27/49], Ahmed 50\% [N=9/18], Molteno 40\% [N=2/5]).

By univariate analysis, underlying heart disease was found to be significantly associated with the presence of HP $(p=0.040)$. Ischemic coronary conditions were found in five out of eight patients, arrhythmia in two patients and valvular heart disease in one patient. The heart disease patients had been using either dual anti-platelet or aspirin dosage of more than $81 \mathrm{mg}$. No statistical significance was found in terms of sex, pre-operative IOP, eye laterality, glaucoma diagnosis, previous glaucoma surgery or pre-operative number of
Table I Baseline characteristics

\begin{tabular}{|c|c|c|}
\hline $\mathbf{N}=72$ & $\mathbf{n}$ & $\%$ \\
\hline Age (years): mean $\pm S D$ & \multicolumn{2}{|c|}{$\begin{array}{l}54.75 \pm 17.65 \\
\text { years }\end{array}$} \\
\hline Sex (female) & 29 & 40.28 \\
\hline \multicolumn{3}{|l|}{ Underlying disease } \\
\hline Diabetes mellitus & 18 & 25 \\
\hline Hypertension & 25 & 34.72 \\
\hline Dyslipidemia & 14 & 19.44 \\
\hline Heart disease & 8 & 11.11 \\
\hline Laterality (right eye) & 35 & 48.61 \\
\hline \multicolumn{3}{|l|}{ Diagnosis } \\
\hline POAG & 10 & 13.89 \\
\hline PACG & 7 & 9.72 \\
\hline Childhood glaucoma & 4 & 5.56 \\
\hline Secondary glaucoma & 51 & 70.83 \\
\hline Secondary glaucoma (post-corneal transplant) & 15 & 20.83 \\
\hline NVG & 12 & 16.67 \\
\hline Unspecified secondary glaucoma & 24 & 33.33 \\
\hline \multicolumn{3}{|l|}{ Pre-operative VA } \\
\hline Equal or better than $20 / 70$ & 16 & 22.22 \\
\hline $20 / 70>V A \geq 20 / 200$ & 7 & 9.72 \\
\hline Worse than $20 / 200$ & 49 & 68.05 \\
\hline Pre-operative IOP: mean \pm SD & \multicolumn{2}{|c|}{$\mathrm{mmHg}$} \\
\hline \multicolumn{3}{|l|}{ Lens } \\
\hline Phakic & 23 & 31.94 \\
\hline Pseudophakic & 43 & 59.72 \\
\hline Aphakic & 6 & 8.33 \\
\hline \multicolumn{3}{|l|}{ Previous glaucoma surgery } \\
\hline 0 & 23 & 31.94 \\
\hline $\mathrm{I}-2$ & 45 & 62.5 \\
\hline $3-4$ & 4 & 5.56 \\
\hline $\begin{array}{l}\text { Pre-operative number of anti-glaucoma } \\
\text { medication: median (IQR) }\end{array}$ & \multicolumn{2}{|c|}{$4(3-4)$} \\
\hline \multicolumn{3}{|l|}{ Type of GDD } \\
\hline Valve & 18 & 25.00 \\
\hline Non-valve & 54 & 75 \\
\hline
\end{tabular}

Notes: Valve GDD = Ahmed; non-valve GDD = Molteno and Baerveldt.

Abbreviations: POAG, primary open angle glaucoma; PACG, primary angle closure glaucoma; NVG, non-valve glaucoma drainage device; VA, visual acuity; IOP, intraocular pressure; GDD, glaucoma drainage device.

medications by univariate analysis. $(p=0.530,0.530,0.803$, $0.477,0.731$ and 0.788 , respectively). Multivariate analysis was performed for which age, underlying dyslipidemia, heart disease, pre-operative VA, lens status and type of GDD were adjusted. The result showed that underlying heart disease was a significant protective factor $(p=0.027$, OR $0.06 ; 95 \%$ CI $0.00-0.72$ ), and pre-operative VA equal or better than $20 / 70$ was a significant risk factor $(p=0.021$, OR $7.5 ; 95 \%$ CI 1.4-41.4) (Table 2).

The overall mean of IOP was significantly lower: $27.1 \pm 9.56 \mathrm{mmHg}$ at pre-operation and $13.67 \pm 5.91 \mathrm{mmHg}$ at 12 -month follow-up $(p<0.001)$. There was a significant reduction in the number of medications used from baseline 
Table 2 Factors related to HP after GDD implantation

\begin{tabular}{|c|c|c|c|c|c|c|c|c|}
\hline & Non-HP & $\mathbf{H P}$ & Crude & $95 \% \mathrm{Cl}$ & $p$-value & Adjusted & $95 \% \mathrm{Cl}$ & $p$-value \\
\hline & n (\%) & n (\%) & odds ratio & & & odds ratio & & \\
\hline Age (years) & $58.68 \pm 18.55$ & $51.24 \pm 16.24$ & 0.98 & $0.95-1.00$ & 0.077 & 0.98 & $0.94-1.01$ & 0.182 \\
\hline \multicolumn{9}{|l|}{ Dyslipidemia } \\
\hline Yes & $9(64.29)$ & $5(35.7 \mathrm{I})$ & 0.42 & $0.13-1.41$ & 0.161 & 0.45 & $0.1-2.05$ & 0.301 \\
\hline No & $25(43.10)$ & $33(56.90)$ & I & & & I & & \\
\hline \multicolumn{9}{|l|}{ Heart disease } \\
\hline Yes & $7(87.50)$ & $\mathrm{I}(12.50)$ & 0.10 & $0.01-0.90$ & 0.040 & 0.06 & $0-0.72$ & 0.027 \\
\hline No & 27 (42.19) & 37 (57.8I) & 1 & & & I & & \\
\hline \multicolumn{9}{|l|}{ Pre-operative VA } \\
\hline Equal or better than $20 / 70$ & $4(25.00)$ & $12(75.00)$ & 3.39 & $0.96-12.00$ & 0.058 & 7.5 & $1.35-4 \mid .35$ & 0.021 \\
\hline $20 / 70>V A \geq 20 / 200$ & $4(57.14)$ & $3(42.86)$ & 0.85 & $0.17-4.19$ & 0.840 & I.31 & $0.21-8.13$ & 0.775 \\
\hline Worse than $20 / 200$ & $26(53.06)$ & $23(46.94)$ & I & & & $\mathrm{I}$ & & \\
\hline \multicolumn{9}{|l|}{ Lens } \\
\hline Phakic & $9(39.13)$ & $14(60.87)$ & 7.78 & $0.78-77.93$ & 0.081 & 3.64 & $0.30-43.96$ & 0.310 \\
\hline Pseudophakic & $20(46.5 I)$ & $23(53.49)$ & 5.75 & $0.62-53.43$ & 0.124 & 6.66 & $0.56-79.85$ & 0.135 \\
\hline Aphakic & $5(83.33)$ & I (I6.67) & I & & & I & & \\
\hline \multicolumn{9}{|l|}{ Type of GDD } \\
\hline Non-valve & $25(46.30)$ & $29(53.70)$ & 1.16 & $0.40-3.37$ & 0.785 & 1.17 & $0.33-4.09$ & 0.81 \\
\hline Valve & $9(50.00)$ & $9(50.00)$ & 1 & & & 1 & & \\
\hline
\end{tabular}

Notes: Valve GDD = Ahmed; non-valve GDD = Molteno and Baerveldt. Multiple logistic regression. Significance $(p<0.05)$.

Abbreviations: HP, hypertensive phase; GDD, glaucoma drainage device; VA, visual acuity.

median (IQR) $4(2-6)$ to $1(0-4)$ at 12 months post-operation $(p<0.001)$; however, no significant improvement in VA after surgery was found in this study ( $p=0.343$ ) (Table 3 ). After GDD implantation, significant difference of mean IOP between HP group and non-HP group was observed only at 2 weeks to 2 months (Figure 3). During the high IOP period, anti-glaucoma medication was adjusted according to the patient's glaucoma status and we found that anti-glaucoma medications were significantly higher in HP group than in non-HP group from 1 month to 4 months after surgery, but no significant difference was observed for IOP and number of medications between the two groups at 1 year post-operation ( $p=0.823$ and 0.138 , respectively) (Table 4$)$. Of all subjects (72 eyes), 26 eyes (36.11\%), 11 eyes in HP group (38 eyes) and 15 eyes in non-HP group ( 34 eyes), did not need antiglaucoma medication at 1 year post-operation.
Early complications were observed in 28 cases (38.9\%) and late complications were observed in 10 cases (13.9\%). The most common early complication was hypotony $(\mathrm{N}=14$, $19.44 \%$ ), whereas persistent corneal edema ( $\mathrm{N}=4,5.55 \%)$ was the most common late complication. In total, 13/28 eyes had more than one complication. Two cases of Baerveldt implantation required removal, both of which were from non-HP group. One was removed at 3 months after surgery due to GDD exposure. The other one had overdrainage, resulting in hypotony maculopathy, wherein GDD was finally removed at 1 month after failed multiple interventions. Failure rate at 12 months was $24 / 72(33 \%)$. VA became no light perception in two out of 24 cases (one from HP and one from non-HP group). Both of them had extremely low pre-operative VA (hand motion) prior to Baerveldt implantation. There was no difference in all surgical outcomes between the two groups in terms of failure,

Table 3 Pre-operative and 12-month post-operative outcomes

\begin{tabular}{|c|c|c|c|}
\hline & $\begin{array}{l}\text { Pre-operative } \\
\text { outcome }\end{array}$ & $\begin{array}{l}\text { Post-operative } \\
\text { I 2-month outcome }\end{array}$ & $p$-value \\
\hline IOP $(\mathrm{mmHg}):$ mean $\pm \mathrm{SD}$ & $27.1 \pm 9.56$ & $|3.67 \pm 5.9|$ & $<0.00 I^{*}$ \\
\hline Numbers of medications: median (IQR) & $4(3-4)$ & I $(0-2)$ & $<0.00 I^{*}$ \\
\hline VA (Snellen): n (\%) & & & $0.343^{\#}$ \\
\hline Equal or better than $20 / 70$ & $16(22.22)$ & $13(18.05)$ & \\
\hline $20 / 70>V A \geq 20 / 200$ & $7(9.72)$ & $12(16.67)$ & \\
\hline Worse than $20 / 200$ & $49(68.05)$ & $47(65.28)$ & \\
\hline
\end{tabular}

Notes: *Wilcoxon signed ranks test compared with pre-operation. \#McNemar Test. Data in bold indicates statistical significance. Abbreviations: IOP, intraocular pressure; VA, visual acuity. 


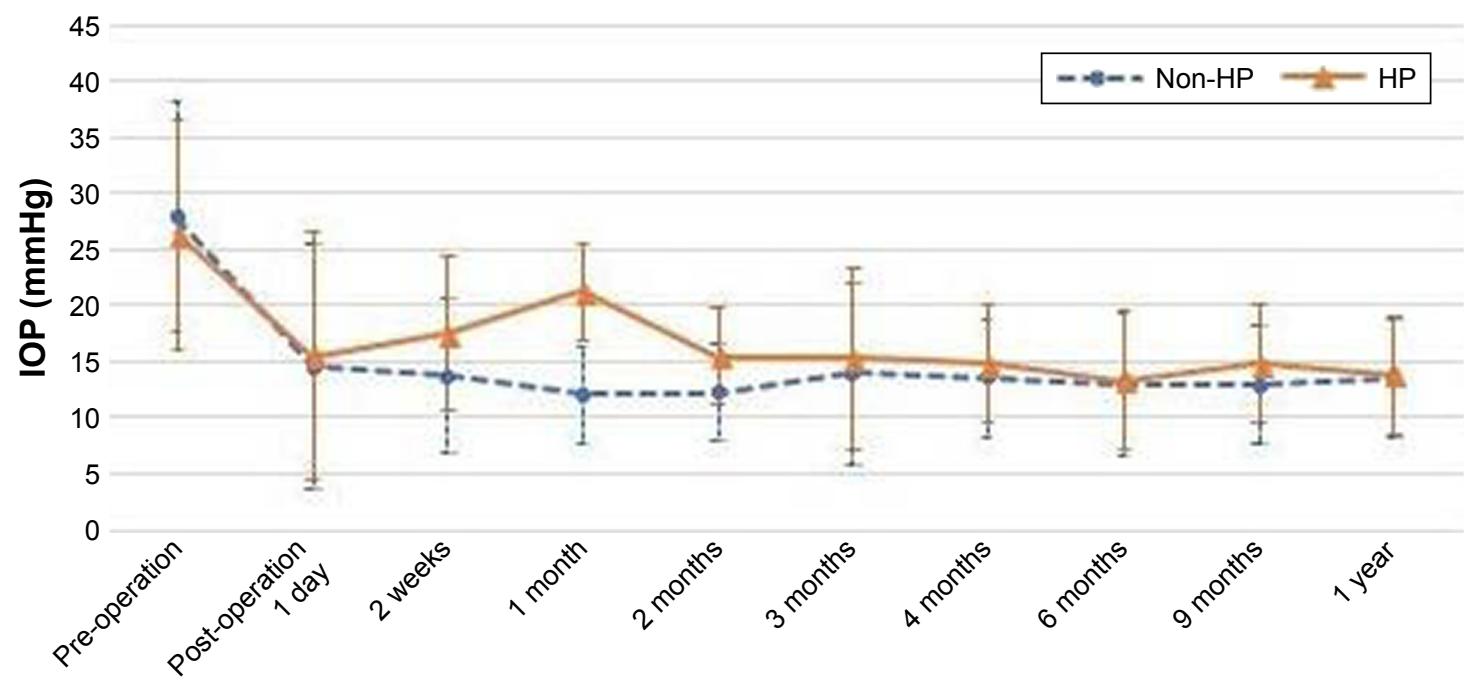

Time

Figure 3 Comparison of IOP after GDD implantation between HP and non-HP groups.

Abbreviations: IOP, intraocular pressure; GDD, glaucoma drainage device; HP, hypertensive phase.

early complication and late complication. The post-operative outcomes are summarized in Table 5. Survival analysis also showed no significant difference between HP group and non-HP group (Figure 4). Factors associated with the failure rate of the GDD implantation were not found in this study.

\section{Discussion}

After GDD implantation, hypotensive phase was observed in the first 7-10 days. IOP gradually increased afterward when the bleb became well circumscribed and intensely congested. The bleb congestion and inflammation decreased over the ensuing months and then the capsule became less dense and IOP subsequently stabilized. ${ }^{3,6,10}$

Table 4 Course of IOP after GDD implantation

\begin{tabular}{lllll}
\hline & Non-HP & & HP & P-value \\
\cline { 2 - 2 } & Mean $\pm \mathbf{S D}$ & & Mean $\pm \mathbf{S D}$ & \\
\hline Pre-operation & $27.85 \pm 10.26$ & & $26.42 \pm 8.97$ & 0.530 \\
Post-op I day & $14.56 \pm 11.00$ & & $15.47 \pm 9.67$ & 0.708 \\
Post-op 2 weeks & $13.74 \pm 6.96$ & & $17.50 \pm 7.70$ & $\mathbf{0 . 0 3 4}$ \\
Post-op I month & $12.06 \pm 4.37$ & & $21.21 \pm 8.4 \mid$ & $<\mathbf{0 . 0 0 1}$ \\
Post-op 2 months & $12.18 \pm 4.29$ & & $15.45 \pm 8.29$ & $\mathbf{0 . 0 3 7}$ \\
Post-op 3 months & $13.91 \pm 8.01$ & & $15.26 \pm 8.99$ & 0.505 \\
Post-op 4 months & $13.62 \pm 5.27$ & & $14.76 \pm 7.43$ & 0.458 \\
Post-op 6 months & $13.03 \pm 6.28$ & & $13.32 \pm 6.61$ & 0.852 \\
Post-op 9 months & $12.91 \pm 5.23$ & & $14.76 \pm 7.88$ & 0.250 \\
Post-op I year & $13.50 \pm 5.28$ & & $13.82 \pm 6.49$ & 0.823 \\
p-value & $\mathbf{0 . 0 8 5}$ & & \\
\hline
\end{tabular}

Notes: Independent $t$-test. Generalized linear mixed model adjusted baseline: age, sex, heart disease, pre-operative VA and type of GDD. Significance $(p<0.05)$. Data in bold indicates statistical significance.

Abbreviations: IOP, intraocular pressure; GDD, glaucoma drainage device; HP, hypertensive phase; VA, visual acuity; Post-op, post-operation.
HP has been reported in both valve and non-valve GDD implantation. Ayyala et al reported that HP was more frequently seen after Ahmed implantation (incidence $40 \%-80 \%)$ than non-valve GDD like Baerveldt (20\%-30\%) or double-plate Molteno implantation (20\%-30\%). ${ }^{2,4}$ It was speculated that this incidence may be related to early contact of glaucomatous aqueous with the surrounding conjunctiva and Tenon's capsule over the device plate. ${ }^{6,10,11}$ The mediators in glaucomatous aqueous such as prostaglandins, various eicosanoids and transforming growth factor $\beta$ can induce inflammatory reaction and result in severe inflammation, fibrosis and poor bleb function. ${ }^{10,12}$ However, the exact pathogenesis is still not clearly understood. ${ }^{3}$

This study revealed the incidence of HP in 38/72 patients (52.8\%, 95\% CI 40.7-64.7): Ahmed 50\%, Baerveldt 55.1\% and Molteno $40 \%$. The rate of HP in Ahmed was the same as shown in the report by Nouri-Mahdavi and Caprioli, but different from that of Ayyala et al's study, perhaps due to different criteria. ${ }^{4,6}$

Table 5 Post-operative outcomes

\begin{tabular}{|c|c|c|c|c|}
\hline & $\begin{array}{l}\text { Non-HP } \\
(n=34) \\
\end{array}$ & $\begin{array}{l}\text { HP } \\
(n=38)\end{array}$ & $p$-value & $p$-value** \\
\hline & n (\%) & n (\%) & & \\
\hline Failure & $12(50.00)$ & $12(50.00)$ & 0.738 & 0.638 \\
\hline Early complication & $15(51.72)$ & $14(48.28)$ & 0.530 & 0.701 \\
\hline Late complication & $3(30.00)$ & $7(70.00)$ & $0.316^{\#}$ & 0.078 \\
\hline
\end{tabular}

Notes: \#Fisher's exact test. **Logistic regression adjusted-baseline: age, sex, heart disease, pre-operative VA and type of GDD. Significance $(p<0.05)$.

Abbreviations: HP, hypertensive phase; VA, visual acuity; GDD, glaucoma drainage device. 


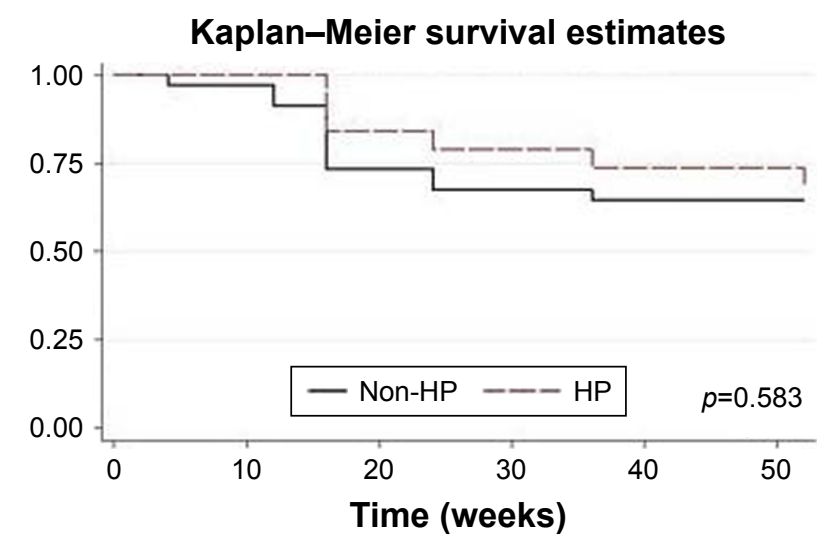

Figure 4 Kaplan-Meier survival curve comparing non-HP and HP group. Abbreviation: HP, hypertensive phase.

In cases with a hypertensive period, IOP markedly declined in the first 2 weeks after surgery, then increased to above $21 \mathrm{mmHg}$ (peak was commonly noted at 1 month) and then gradually dropped to a steady phase. During the hypertensive phase, IOP could be as high as $50 \mathrm{mmHg}$, which can significantly affect the vulnerable retinal nerve fiber layers. Therefore, need of careful early post-operative follow-up for patients at risk after drainage device implantation is recommended, for necessary rescue.

Both HP and non-HP groups had significant reduction in IOP and number of medications from baseline, with no significant difference between both groups at 12-month follow-up. The main treatment of HP was controlled by medication, which could be tailed off thereafter in most patients. At the 12-month follow-up period, 11/38 patients of HP group did not need the anti-glaucoma medication to control IOP.

With regard to factors related to HP in this study, it was interesting that the presence of underlying heart disease was found as a protective factor. Patients with heart disease had long-term usage of aspirin dosage of more than $81 \mathrm{mg}$ or dual anti-platelet treatment (aspirin combined with other anti-platelet drug) before and after GDD implantation; the possibility of this connection may be from the effect of the anti-inflammatory action of aspirin that helped control inflammation and promote implant function. ${ }^{13,14}$ The previous report of Molteno et al had evaluated the anti-inflammatory agents and reported aspirin as a moderately active agent that could lower IOP by $5-10 \mathrm{mmHg}$, and proposed the effectiveness of a triple anti-inflammatory drug regimen, consisting of oral prednisolone, flufenamic acid and topical l-adrenaline, in controlling bleb inflammation and fibrosis around drainage implants and promoting implant function. ${ }^{15}$ However, due to the small group of patients with heart disease and limitation of the retrospective study, further evaluation of the effect of aspirin and other anti-inflammatory agents in relation to HP is warranted.

Another factor associated with the development of HP was pre-operative VA equal to or better than 20/70, identified as a risk. It was speculated that these eyes might have had earlier GDD intervention because of the aggressiveness of their uncontrolled IOP condition rather than severity of optic nerve head status, which might be represented by worse central VA. The unmanageable IOP control conditions may in turn reflect their stronger inflammatory mediators and tissue responses to the intervention where leading to higher chance of HP. This speculation needs to be proven. In addition, neither type of GDD nor pre-operative IOP was found to be associated with the development of HP. High myopia and higher pre-operative IOP were reported to be the risk factors of HP after Ahmed implantation in previous studies. ${ }^{8,9}$ However, our results were different from those reported; this could be due to the presence of different factors and the fact that our studied GDD was not restricted to Ahmed.

The complications after GDD implantation were comparable between HP and non-HP groups. Most of the complications were spontaneously resolved without further intervention, except ones with exposed plate and exposed tube, which required subsequent GDD removal. The latter was inevitably considered as surgical failure. The improvement of post-operative VA was reported as $87.4 \%$ after Baerveldt implantation by Siegner et al. ${ }^{16}$ However, there was no significant improvement in this study, which was also not found in studies of Ayyala et al. ${ }^{2,4}$ In addition, there was no difference in all surgical outcomes between the two groups. Survival analysis also showed no significant difference between HP and non-HP groups. This study could not identify the factors associated with the failure rate of the GDD implantation.

The main limitations of this study included its retrospective design and small sample size. There was also a limitation in terms of applying the definition of HP, which varies among studies; most studies defined HP as IOP more than $21 \mathrm{mmHg}$ during the first 3 months after surgery with or without medications. This criterion suited HP evaluation in valve GDD. With the tube ligation technique in non-valve GDD implantation, despite that venting slits were performed and able to lower IOP particularly in the first week post-operation, the IOP usually did not decrease considerably compared to immediate post-operative IOP in valve GDD. Later, the IOP became relatively high and was sustained until the ligating material degraded, which usually occurred $4-8$ weeks after surgery. Considering this pattern of IOP change, we decided 
to add another criterion, which was IOP $>21 \mathrm{mmHg}$ after 2 consecutive weeks of the functional post-operative period, marked as noticeably lower IOP than $22 \mathrm{mmHg}$, to avoid the overestimation of the rate of HP in this study.

\section{Conclusion}

GDD was an effective surgical procedure to control the IOP and reduce the use of medications in intractable glaucoma. HP commonly occurred after GDD implantation with a similar rate in both valve and non-valve GDD. Pre-operative VA was a risk factor for the development of HP, whereas presence of underlying heart disease was a protective one. Device type did not influence HP incidence. No association between HP and surgical outcomes was identified.

\section{Author contributions}

All the authors were involved in the following:

1) Contributions to conception and design, acquisition of data, and analysis and interpretation of data

2) Drafting the article or revising it critically for important intellectual content

3) Final approval of the version to be published

4) Agreement to be accountable for all aspects of the work in ensuring that questions related to the accuracy or integrity of any part of the work are appropriately investigated and resolved.

\section{Disclosure}

The authors report no conflicts of interest in this work.

\section{References}

1. Molteno AC, Dempster AG. Methods of controlling bleb fibrosis around draining implants. In: Mills KB, editor. Glaucoma: proceedings of the Fourth International Symposium of the Northern Eye Institute, Manchester, UK, 14-16 July 1988. 1st ed. Oxford; New York: Pergamon Press; 1989:192-211.
2. Ayyala RS, Zurakowski D, Smith JA, et al. A clinical study of the Ahmed glaucoma valve implant in advanced glaucoma. Ophthalmology. 1998;105(10):1968-1976.

3. Kawai M, Yamaguchi T, Nakabayashi S, Yoshida A. Combined Baerveldt glaucoma drainage implant surgery and surgical bleb revision for preventing a postoperative hypertensive phase. Clin Ophthalmol. 2014;8:773-776.

4. Ayyala RS, Duarte JL, Sahiner N. Glaucoma drainage devices: state of the art. Expert Rev Med Devices. 2006;3(4):509-521.

5. Ayyala RS, Zurakowski D, Monshizadeh R, et al. Comparison of double-plate Molteno and Ahmed glaucoma valve in patients with advanced uncontrolled glaucoma. Ophthalmic Surg Lasers. 2002;33(2): 94-101.

6. Nouri-Mahdavi K, Caprioli J. Evaluation of the hypertensive phase after insertion of the Ahmed Glaucoma Valve. Am J Ophthalmol. 2003; 136(6):1001-1008.

7. Christakis PG, Tsai JC, Kalenak JW, et al. The Ahmed versus Baerveldt study: three-year treatment outcomes. Ophthalmology. 2013;120(11): 2232-2240.

8. Jung KI, Park CK. Risk factors for the hypertensive phase after implantation of a glaucoma drainage device. Acta Ophthalmol. 2016;94(5): e260-e267.

9. Won HJ, Sung KR. Hypertensive phase following silicone plate Ahmed glaucoma valve implantation. J Glaucoma. 2016;25(4):e313-e317.

10. Pakravan M, Rad SS, Yazdani S, Ghahari E, Yaseri M. Effect of early treatment with aqueous suppressants on Ahmed glaucoma valve implantation outcomes. Ophthalmology. 2014;121(9):1693-1698.

11. Law SK, Kornmann HL, Giaconi JA, Kwong A, Tran E, Caprioli J. Early aqueous suppressant therapy on hypertensive phase following glaucoma drainage device procedure: a randomized prospective trial. J Glaucoma. 2016;25(3):248-257.

12. Tripathi RC, Li J, Chan WF, Tripathi BJ. Aqueous humor in glaucomatous eyes contains an increased level of TGF-beta 2. Exp Eye Res. 1994;59(6):723-727.

13. Amann R, Peskar BA. Anti-inflammatory effects of aspirin and sodium salicylate. Eur J Pharmacol. 2002;447(1):1-9.

14. Yin MJ, Yamamoto Y, Gaynor RB. The anti-inflammatory agents aspirin and salicylate inhibit the activity of I(kappa)B kinase-beta. Nature. 1998;396(6706):77-80.

15. Molteno AC, Straughan JL, Ancker E. Control of bleb fibrosis after glaucoma surgery by anti-inflammatory agents. S Afr Med J. 1976;50(23): $881-885$.

16. Siegner SW, Netland PA, Urban RC Jr, et al. Clinical experience with the Baerveldt glaucoma drainage implant. Ophthalmology. 1995;102(9): $1298-1307$.
Clinical Ophthalmology

\section{Publish your work in this journal}

Clinical Ophthalmology is an international, peer-reviewed journal covering all subspecialties within ophthalmology. Key topics include: Optometry; Visual science; Pharmacology and drug therapy in eye diseases; Basic Sciences; Primary and Secondary eye care; Patient Safety and Quality of Care Improvements. This journal is indexed on Submit your manuscript here: http://www.dovepress.com/clinical-ophthalmology-journal

\section{Dovepress}

PubMed Central and CAS, and is the official journal of The Society of Clinical Ophthalmology (SCO). The manuscript management system is completely online and includes a very quick and fair peer-review system, which is all easy to use. Visit http://www.dovepress.com/ testimonials.php to read real quotes from published authors. 